\title{
LOS ROLES DE LOS ACTORES DE LA SOCIEDAD DE ACOGIDA EN LA INTEGRACIÓN DE LAS ASOCIACIONES DE INMIGRANTES: UNA APROXIMACIÓN DESDE EL ANÁLISIS DE REDES SOCIALES ${ }^{1}$
}

\section{THE ROLES OF ACTORS IN THE HOST SOCIETY IN THE INTEGRATION OF IMMIGRANTS ASSOCIATIONS: A SOCIAL NETWORKS ANALYSIS APPROACH}

José Manuel Gaete Fiscella* y Luis Mena Martínez**

\begin{abstract}
Resumen: Este artículo indaga sobre los estadios de integración de las asociaciones de inmigrantes en España según el stock de capital social que detentan, específicamente, dadas las relaciones que establecen con actores del entorno, y consecuentemente el rol que de esto se deriva para cada tipo de contraparte. Los datos se extraen de una muestra representativa conformada por 225 asociaciones de inmigrantes de todo el territorio español. Los resultados apuntan a que las asociaciones de inmigrantes juegan roles especificos y cohesivos con sus pares, incluso logran ciertas asimetrías en su favor con determinados actores de la sociedad de acogida. Por otro lado, los actores nativos de la sociedad de acogida se distribuyen en un continuo que va desde el desarrollo de roles específicos hasta otros múltiples, e incluso contradictorios, lo que, en su conjunto, termina por dibujar un complejo y diverso mapa de oportunidades para la integración de las asociaciones.
\end{abstract}

Investigador postdoctoral FONDECYT- CEDER. Universidad de Los Lagos, Chile. josemanuel.gaete@ulagos.cl

** Profesor Contratado Doctor, Departamento de sociología y comunicación, Universidad de Salamanca, España. luismena@usal.es

1 Artículo desarrollado en el marco del Proyecto CONICYT-Fondecyt / postdoctorado $\mathrm{N}^{\mathrm{o}} 3150449$ 
Palabras clave: redes sociales, asociaciones, inmigrantes, integración, capital social.

Abstract: This article explores the stages of integration of immigrant associations in Spain according to stock of social capital, specifically, given the relationships established with actors in the environment, and consequently this role is derived for each type of counterpart. The data are drawn from a representative sample consisting of 225 immigrant associations around the country. The results suggest that immigrant associations play cohesive and specific roles with their peers, even achieve certain asymmetries in their favor with certain actors in the host society. On the other hand, native actors host society are distributed in a continuum ranging from the development of specific roles to other multiple, and even contradictory, which, as a whole, ends up drawing a map of complex and diverse opportunities for integration of associations.

Keywords: social networks, associations, immigrants, integration, social capital.

\section{INTRODUCCIÓN}

La emergencia y relevancia del asociacionismo inmigrante extracomunitario en la sociedad española, aunque reciente, se funda y expresa en el actuar de diversos actores.

Por un lado, para los propios colectivos inmigrantes desde la necesidad de desarrollar diversas funciones y satisfacer necesidades: culturales, materiales, identitarias, etc. (Morell, 2005; González y Morales, 2006; Aparicio y Tornos, 2010; Herrera, 2008; Álvarez de los Mozos, 2013)

Por otro lado, los diversos actores españoles también evidencian la relevancia y legitimidad de estas asociaciones, tanto el Estado, como las autonomías, la sociedad civil o el sector privado. El Estado español a través de distintas leyes y decretos (Ley Orgánica B.O.E. 4/2000, Ley Orgánica 1/2002, etc.), sanciona, dentro del proceso de integración social, la definición y actuar de estos actores organizacionales, destinando fondos y subsidios que apoyan la formación y desarrollo de estas organizaciones (Veredas, 2003; Gómez, 2008). En este contexto, es lógico esperar que la mayoría de las comunidades autónomas cuenten con programas y/o planes de integración para este colectivo, y dentro de los cuales se considere la participación de las asociaciones de inmigrantes como representantes válidos de la comunidad inmigrante (Herrera, 2008). 
Desde la sociedad civil, las propias ONG's definen a las asociaciones de inmigrantes como socios estratégicos indispensables para conocer las necesidades y problemas del colectivo inmigrante, y también, como actores partícipes en la definición, implementación y gestión de programas orientados a mejorar la calidad de vida e integración de los propios inmigrantes. Bajo una lógica de cooperación similar, el sector privado también ha reaccionado frente a la aparición de estas organizaciones al considerarloscomo socios idóneos que permiten la concreción de las estrategias de marketing social o la responsabilidad social corporativa (Rodríguez y Carrasco, 2003).

Además de las referencias anteriores, es preciso constatar que este fenómeno asociativo también cobra relevancia propia a nivel agregado. De acuerdo a los datos que proporciona tanto el registro nacional de asociaciones de España como los registros autonómicos es posible constatar cómo éste fenómeno presenta una creciente relevancia, tal y como se evidencia en el siguiente gráfico.

FIgURA 1

EVOLUCIÓN DE LA INSCRIPCIÓN DE ASOCIACIONES

DE INMIGRANTES EN LOS REGISTROS OFICIALES AUTONÓMICOS Y NACIO_NALES

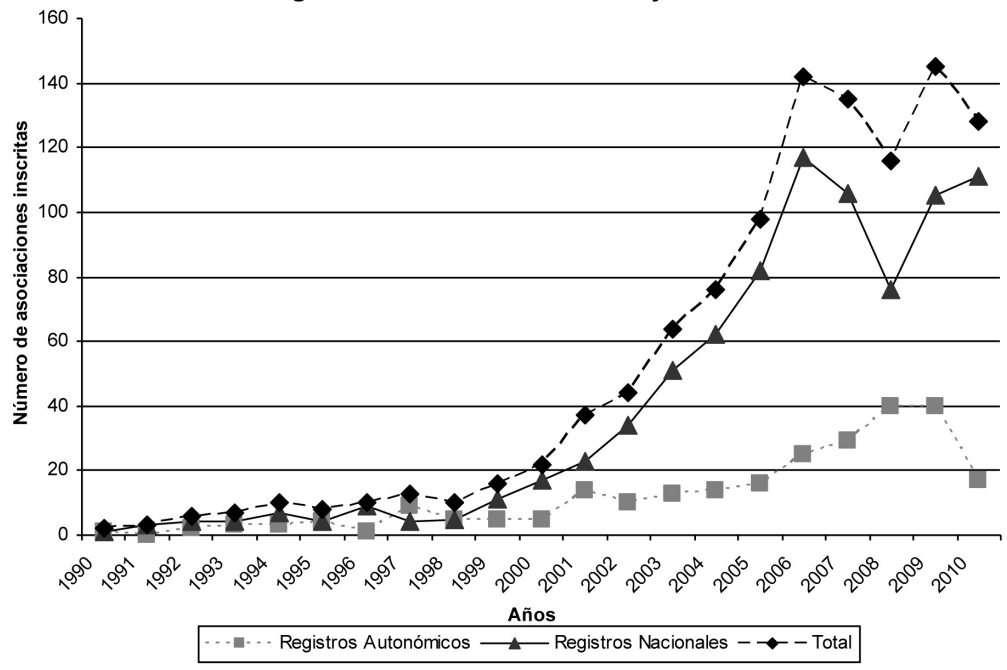

Fuente: elaboración propia según los registros entregados por el Ministerio del Interior de España y de las Comunidades Autónomas (2011). 
Como se puede observar en el gráfico 1, el aumento de asociaciones de inmigrantes se produjo de manera considerable a partir del año 1999 para los registros nacionales, y algo más tarde en los autonómicos ${ }^{2}$, obteniéndose el número máximo en el año 2007 para los primeros y el 2008 y 2009 para los segundos ${ }^{3}$.

Además de lo anterior, parece difícil poner en duda la relevancia del fenómeno asociativo si consideramos que la irrupción de estas asociaciones puede ser considerado como la emergencia de un sujeto inmigrante de naturaleza organizacional a nivel mesosocial (Lazega, 2004). Por tanto, y además de preguntarse cómo (o cuánto) se integran estos actores en la sociedad de acogida, también surge la pregunta por el papel o rol que cumple la sociedad de acogida en este proceso, particularmente los actores nativos que con ellas se relacionan e interactúan, pregunta que es fundamental en este trabajo.

\section{MARCO TEÓRICO}

\subsection{Relaciones y redes interorganizativas}

La cuestión de la integración inmigrante nunca ha sido una cosa fácil de plantearse para las realidades microsociales, y menos aún para las mesosociales donde existen más preguntas que certezas. Así, en las recientes y escasas investigaciones realizadas sobre la integración de asociaciones de inmigrantes en la sociedad española encontramos concepciones y referencias a la integración en términos relacionales. Por ejemplo, en el trabajo de Morell (2005) la integración se define bajo concepciones de cooperación entre las asociaciones y los actores de la sociedad de acogida, sobre todo a la hora de intermediar en los procesos de asistencia y ayuda que la sociedad de acogida oferta a la población inmigrante a través de actores tanto públicos como privados. En el ámbito político, la integración de las asociaciones inmigrantes parece jugarse bajo una lógica y expresión relacional similar, esto es, según el grado o

2 La base de comparación de los registros autonómicos es mucho menor dado que en gran parte de los registros no se explicita el año de fundación de las asociaciones.

3 Los registros de las asociaciones fueron solicitados en el 2012, por lo que las reparticiones autonómicas hicieron llegar los registros que contabilizaban hasta el año 2011. 
nivel de participación política que dichas organizaciones alcanzan en la sociedad española (González y Morales, 2006), y operacionalizado en función de la participación en actividades políticas tales como contactos con organizaciones políticas y autoridades de la sociedad de acogida, también como la frecuencia con la que participan en los procesos de toma de decisiones mediante el acceso y participación en las instancias consultivas o decisionales. En la misma línea, para Tillie (2004), la integración política de los inmigrantes a nivel mesosocial está dada por la frecuencia y densidad de relaciones cooperativas que se recreen entre las propias asociaciones inmigrantes y los actores nativos, sean estos públicos o privados (Cheng, 2005).

No obstante lo anterior, las referencias a las relaciones entre asociaciones y actores del entorno parecen diversas y acaso ambiguas, por tanto, y como punto inicial, cabe preguntarse qué se entiende por las relaciones entre las asociaciones y los actores del entorno. Para este trabajo hemos decidido tomar como base o mínimo común relacional los lazos más débiles que se pueden definir dentro de las relaciones interorganizativas, específicamente nos referimos a las relaciones de cooperación puntual, esto es, interacciones informales o formales con probable ausencia de objetivos y propósitos a largo plazo, y donde tanto la autoridad como los recursos son una prerrogativa y dominio de cada miembro de la interacción, y que asoman como base mínima y suficiente para evaluar las relaciones integradoras (Gajda, 2004; Frey et al., 2006). Además de su naturaleza integradora, estas relaciones de amplio espectro (cooperación puntual/lazos débiles), permiten incluir relaciones intragrupales, pero sobre todo, abrirse a considerar las intergrupales (generalmente entre nativos e inmigrantes); de igual forma, tampoco se excluyen las relaciones más densas o fuertes que pudieran desarrollarse entre los interactuantes, al contrario, una base relacional tan amplia nos permite acceder a una mayor variedad y número de interacciones entre las asociaciones y actores del entorno. Por último, y desde lo metodológico, las relaciones interorganizativas así definidas nos permiten llegar a los respondientes con un concepto más amplio, facilitando así el dar cuenta de las diversas actividades relacionales que puede desarrollar una asociación.

\subsection{Capital Social: bases de la integración social}

Ahora bien, el stock de relaciones interorganizativas, y la red que en su sumatoria se conforma entre las asociaciones de inmigrantes 
y los actores del entorno, siempre bajo lógicas cooperativas, tiene un sustento teórico desde el paradigma del capital social. Así, Coleman (1990), contempla el capital social como un bien colectivo presente tanto en el ámbito grupal como personal, y que se relaciona con el grado de integración social de un individuo, todo a partir de su red de contactos, relaciones, expectativas de reciprocidad, etc. Algo que se alinea con lo planteado por Putnam (2001), para quien el capital social, a grandes rasgos, se define en términos de asociacionismo, confianza y formación de redes cívicas. En la misma línea, y desde lo más específicamente reticular, el trabajo exploratorio de Bolíbar (2010) también correlaciona los stocks de relaciones interorganizativas de las asociaciones de inmigrantes en España con estadios y formas de integración social, y más específicamente, analiza cómo ciertos indicadores estructurales y reticulares dan cuenta de los diferenciales de integración. Más allá de las particularidades que cada autor plantea respecto del capital social, están las regularidades conceptuales que nos hablan de los pilares o bases que fundamentan la complejidad del capital social; y que Putnam (2001) sintetiza y resume cuando afirma que el capital social emerge como los aspectos de las organizaciones sociales, tales como las redes, las normas y la confianza que permiten la acción y la cooperación para el beneficio mutuo (Portela y Neira, 2002).

Las relaciones entre los sujetos (sean personas, organizaciones, etc.) no siempre se plantean bajo lógicas de cooperación, simetría y horizontalidad. Tal como lo plantea Putnam (2001) cuandoconsidera que las relaciones pueden ser definidas como verticales en tanto ligan a los interactuantes a través de mecanismos de autoridad, clientelismo o poder, incluso de explotación, y que devienen en posiciones desiguales entre los interactuantes, y con ello, la reafirmación de la consiguiente estratificación social (Smith, 2003; Kärrholm, 2007; Garnica et al., 2004; Arcas y Hernández, 2007; Levine y White, 1961; Álvarez de los Mozos, 2013). En contraposición, las relaciones horizontales se definen a partir del carácter simétrico de la relación, esto es, relaciones con ausencia de diferenciales de poder y dominación entre los interactuantes, dando lugar a relaciones de tipo cooperativo en planos de igualdad, independencia y autonomía de recursos y fines. 


\subsection{Relaciones horizontales y verticales: oportunidades y límites del Capital Social}

Para Putnam (2001) las relaciones horizontales son aquellas que se forman en el seno de las asociaciones cívicas, en el tejido asociativo comunitario y, en definitiva, constituyen componentes básicos del capital social (Lozares et al., 2011). Así, la prevalencia de las relaciones horizontales sobre las verticales permite la cohesión y el trabajo conjunto entre los ciudadanos en pos de las soluciones de problemas comunitarios, el desarrollo de la sociedad civil y la integración de los sujetos en la sociedad (Plascencia, 2005), hechos sociales que son inexistentes cuando las relaciones se desarrollan en su modalidad vertical (Sardinha, 2005). De no hacer la diferenciación, tendríamos que asumir que existe una igualdad entre ambos tipos de relaciones, en tanto la horizontalidad y la verticalidad serían propiedades equivalentes y, por ende, propias del capital social y de la integración social, algo que no es exacto, ya que el capital social se construye sólo (o preferentemente) bajo lógicas relacionales simétricas u horizontales (Putnam, 1995; 2011 [1993], Flora, 1998, Ostrom et al., 2003, Mayoux, 2001) dado que la confianza, reciprocidad y cooperación, como atributos básicos de este tipo de capital, se evidencian en grupos cuyas relaciones se fundan en la horizontalidad (World Bank, 1997, Fukuyama, 2001). Tal y como lo afirma el propio Putnam (2011[1993]: 248) Una red vertical, por muy densa e importante que sea para sus participantes, no puede mantener la confianza y la cooperación. En definitiva, cada tipo de relación tiene consecuencias diferentes para la formación de capital social y para las posibilidades y formas de integración de cada sujeto.

Desde lo propuesto por Schul y Babakus (1988) y HernándezEspallardo y Arcas-Lario (2003) planteamos que la definición de las relaciones interorganizativas se fundamenta, básicamente, en considerar el diferencial decisional que puede existir entre las partes interactuantes a la hora de tomar decisiones respecto de la actividad que las convoca, y que se expresa en el grado de participación activa que cada una de las partes tiene en la toma de decisiones respecto de las actividades que fundamentan la relación. Por tanto, será en este indicador de participación decisional relacional desde donde operacionalizaremos nuestra medición sobre el «grado de poder que existe en las relaciones interorganizativas desarrolladas entre las asociaciones de inmigrantes y los actores del entorno». Entendiendo 
que un equilibrio de la participación implica simetría decisional (relaciones horizontales) y, por el contrario, un desequilibrio implica asimetría o el desarrollo de relaciones verticales. Y es a partir de estos tipos de relaciones, dada su combinatoria, volumen y relevancia estructural desde donde definiremos los roles que los actores del entorno juegan en la formación de capital social integrador de las asociaciones de inmigrantes.

\section{MUESTRA Y TÉCNICAS}

\subsection{Muestra}

Los datos se extraen de una encuesta aplicada a una muestra representativa conformada por 225 asociaciones de inmigrantes, de todo el territorio nacional y de todas las regiones de origen extracomunitario (excepto asiáticos). Para esto se definió una muestra estratificada atendiendo a tres variables fundamentales que intervienen los niveles de asociacionismo inmigrante: a) la distribución de la población inmigrante en las distintas comunidades autónomas según el origen o nacionalidad de los mismos (Morales et al., 2008), b) el nivel de concentración de dichos colectivos en las diferentes regiones de España (Fullaondo, 2007) y c) la densidad asociativa de los inmigrantes en cada comunidad autónoma. Entendiendo que el asociacionismo inmigrante depende, entre otros factores, de una densidad mínima de personas inmigrantes en un ámbito como masa crítica que entregue sustento a las asociaciones (Veredas, 2003).

A partir de las cifras de inmigrantes que entrega el INE para el año 2011 hemos definido la proporción de inmigrantes en cada comunidad autónoma; de igual modo hemos considerado los registros de asociaciones a nivel nacional y de las comunidades autónomas, desde lo cual definimos los niveles de proporción de población y densidad asociativa. A partir de estos datos también calculamos el «coeficiente de especialización de absorción» de inmigrantes propuesto por Fullaondo (2007) para cada comunidad autónoma, lo que termina por definir el perfil de cada comunidad en tanto especialización para «absorber»o «acoger» determinados colectivos inmigrantes. Esto último, sumado a las clasificaciones de 


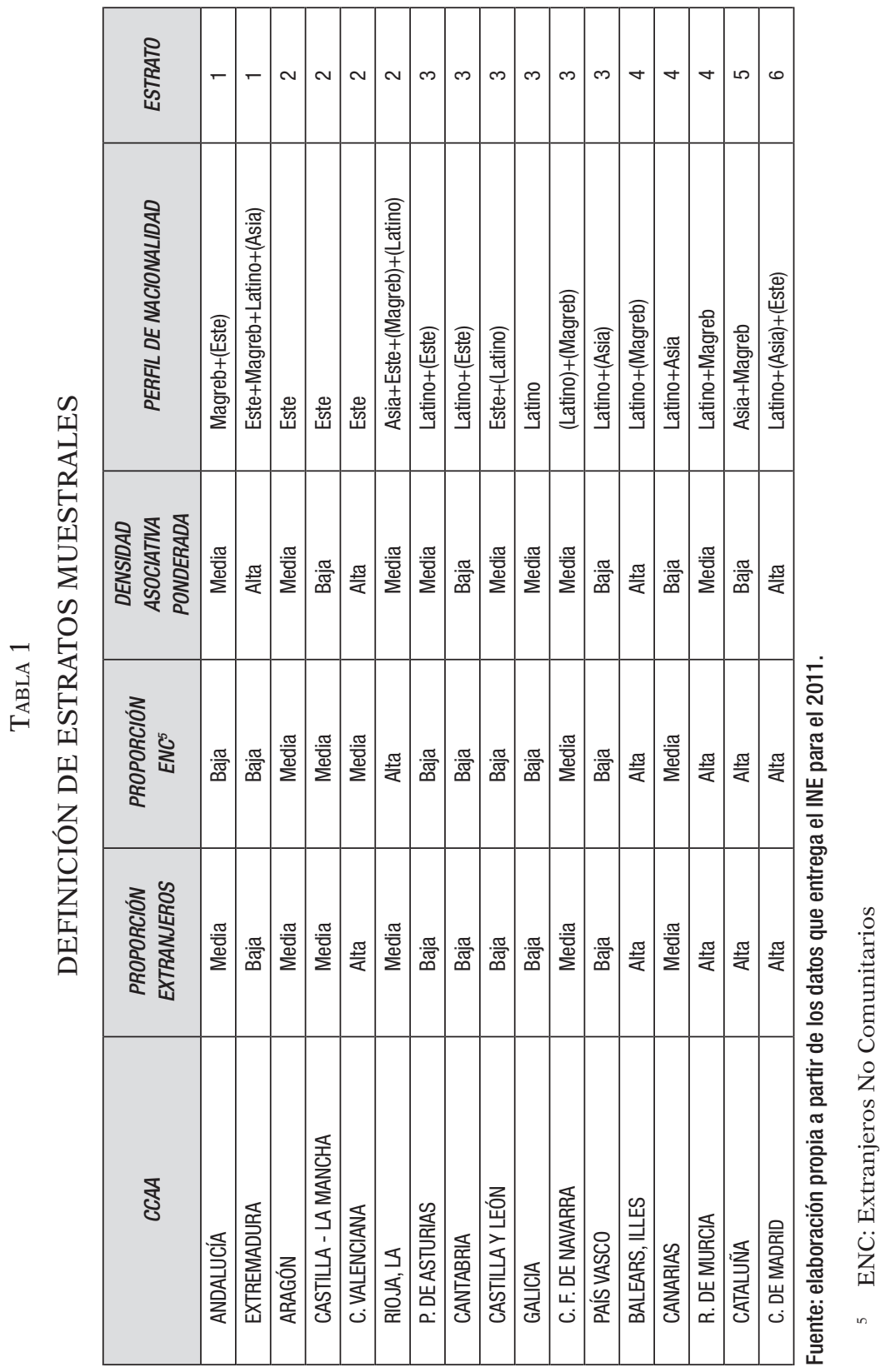


las distribuciones en niveles Bajo, Medio y Alto $^{4}$ nos permite construir los estratos a partir de los cuales se definieron las submuestras, tal y como se explicita en el siguiente cuadro.

Además, la estratificación también sigue un patrón geográfico según el grado de homogeneidad existente entre las comunidades autonómicas; tal y como se presenta en el siguiente figura donde cada uno de los seis estratos se identifica con un tramado y número específico; figura que se acompaña también de la afijación proporcional calculada para cada estrato.

\section{FiguRA 2}

\section{MAPA DE ESPAÑA SEGÚN PERTENENCIA DE LAS COMUNIDADES AUTÓNOMAS A LOS ESTRATOS MAESTRALES}

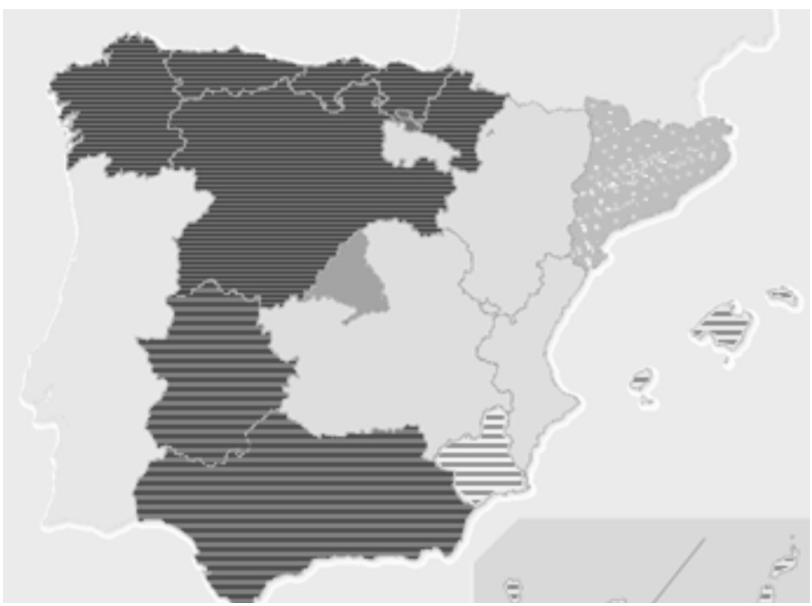

Fuente: elaboración propia.

\begin{tabular}{|l|c|}
\hline \multicolumn{1}{|c|}{ Estrato } & $\begin{array}{c}\text { Afijación } \\
\text { Teórica (Muestra Real) }\end{array}$ \\
\hline -1. Sur-oeste & $45(37)$ \\
\hline -2. Centro-Este & $50(44)$ \\
\hline -3. Nor-oeste & $38(37)$ \\
\hline
\end{tabular}

\begin{tabular}{|l|c|}
\hline \multicolumn{1}{|c|}{ Estrato } & $\begin{array}{c}\text { Afijación } \\
\text { Teórica (Muestra Real) }\end{array}$ \\
\hline -4. Murcia e Islas & $45(38)$ \\
\hline -5. Cataluña & $34(23)$ \\
\hline -6. Madrid & $56(46)$ \\
\hline
\end{tabular}

4 El cálculo se ha efectuado tomando como unidad de desviación la menor proporción de población inmigrante para cada clasificación. Así, el promedio nacional menos la menor proporción define el límite para los niveles denominados como bajos, por el contrario, el promedio nacional más la mínima proporción define el límite superior para definir los niveles altos. 
Cabe consignar que para la afijación proporcional se consideraron dos criterios: la distribución de la población inmigrante y la distribución de las asociaciones en los seis estratos definidos, cifras que, promediadas aritméticamente, definen las cifras finales. Por último, si bien no se logró completar la cuota de la afijación propuesta (teórica) para cada estrato, aún cuando se contactó a la totalidad del marco muestral, la muestra consultada alcanza un error de un $\pm 6,3 \%$ si se considera un muestreo aleatorio simple.

\subsection{Instrumento}

El instrumento de recogida de datos fue una encuesta online (CAWI) dirigida a los responsables de cada asociación. Y las preguntas aplicadas se configuraron en torno a dos dimensiones:

- Tipo de contraparte: si se trata de una entidad de tipo estatal, privada, de la sociedad civil nativa o inmigrante (otras asociaciones de inmigrantes).

Pregunta a.1) Si pensamos en los dos últimos años. ¿Su asociación ha establecido contactos o relaciones con entidades...?

Opciones de respuesta: a) estatales; b) sociedad civil; c) organizaciones inmigrantes; d) entidades comerciales (nombrar un máximo de 5 para cada tipo de contraparte)

- Tipos de relaciones: según el diferencial de poder que se crea en la relación diádica entre las asociaciones y los actores a partir de la capacidad de control de cada una de las partes en las actividades $\mathrm{y}$ acciones que conjuntamente desarrollaron.

Pregunta a.3) Mientras duró la relación. ¿Cuánta influencia tuvo su asociación a la hora de tomar decisiones?

Opciones de respuesta: a) nada, no decidíamos nada; b) poco o muy poco; c) ambas decidíamos por igual; d) mucha o bastante; e) total, decidíamos todo.

A partir de estas preguntas se procedió, por un lado, a definir los tipos de contrapartes con que se relacionaron con las asociaciones de inmigrantes y, por otro, a caracterizar las relaciones con cada tipo de contraparte, todo en función del grado de influencia que tenía tanto la asociación como la contraparte identificada respecto de las actividades que se definieron en la relación. 


\section{RESULTADOS}

Para analizar y definir el rol o papel que los actores nativos juegan en la integración social de las asociaciones inmigrantes se ha optado por utilizar técnicas de análisis de redes sociales, específicamente diseñadas para identificar y medir las relaciones entre los nodos (asociaciones de inmigrantes y actores nativos). En concreto, la estrategia seguida consistió en la construcción y análisis de redes de modo 2 de «asociaciones por actores» (Borgatti y Everett, 1997). Más específicamente, cuatro tipo de redes: una red a partir del número de total de relaciones (TOTAL); una segunda red en que las asociaciones afirman que la contraparte es la que toma todas o la mayoría de las decisiones (NEG); una tercera red a partir de la situación opuesta, esto es, la asociación es la que tiene la mayor influencia (POS) y, finalmente, una cuarta red de relaciones en donde sólo se consideran las relaciones simétricas (SYM) en tanto ambas partes tienen igual capacidad y poder de decisión.

Además de la "centralidad de grado ponderado», en tanto número y frecuencia de relaciones directas entre los nodos-actores (Opsahl et al., 2010) hemos considerado el cálculo de los denominados «valores eigen $»^{5}$ a fin de poder discriminar si el grado de influencia (recibida o ejercida) de los actores se debe más a una centralidad local o, por el contrario, a una centralidad más estructural (Hanneman y Riddle, 2005; Borgatti y Everett, 1997). Además de esto, hemos incluido la «centralidad de intermediación» como tercer indicador, ya que de esta forma podemos evaluar hasta qué punto los distintos tipos de relaciones pueden ser formas de control estructural, principalmente bajo relaciones asimétricas negativas ya que se sumaría a la influencia y control diádico definido por la propia asimetría de la relación. Finalmente, para evidenciar todo esto se presenta una tabla que resume las puntuaciones para todos los tipos de actores según los tipos de relaciones dado los indicadores descritos.

5 El enfoque «eigenvector»o "valor eigen» es un intento por identificar a los actores más centrales (i.e. aquéllos con menor lejanía que otros) en términos de estructura 'global' o 'general' y, con ello, prestando menos atención a patrones más locales (Hanneman y Riddle, 2005). 


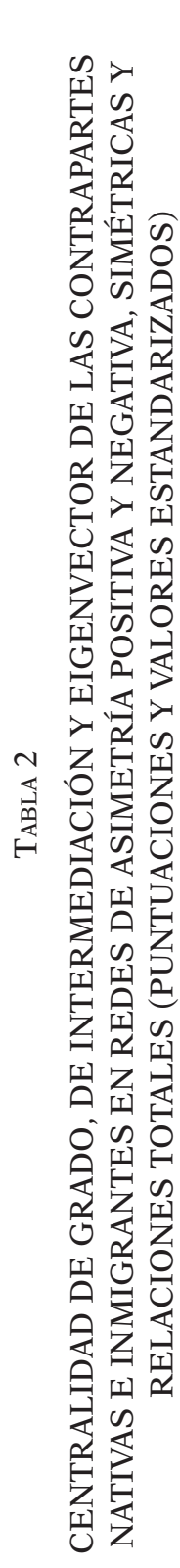

\begin{tabular}{|c|c|c|c|c|c|c|c|c|c|c|c|c|c|c|c|c|c|c|c|c|}
\hline \multirow{4}{*}{ 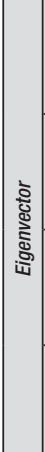 } & 竎 & $\mid \begin{array}{l}\mathbb{\pi} \\
\tilde{0} \\
\dot{0} \\
0 \\
0\end{array}$ & 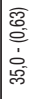 & 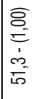 & 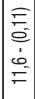 & 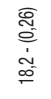 & 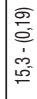 & $=\begin{array}{l}0 \\
\delta \\
0 \\
\dot{0} \\
\dot{c} \\
0\end{array}$ & 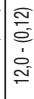 & 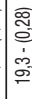 & 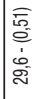 & 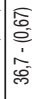 & 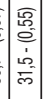 & 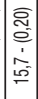 & 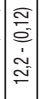 & 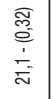 & $\begin{array}{l}\widehat{\bar{b}} \\
0 \\
\dot{0} \\
\dot{0} \\
\tilde{n}\end{array}$ & $\begin{array}{c}E \\
E \\
0 \\
b \\
b \\
E\end{array}$ & $\begin{array}{c}\bar{\sigma} \\
0 \\
0 \\
\dot{0} \\
\infty \\
\infty\end{array}$ & 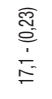 \\
\hline & 2 & \begin{tabular}{|c|} 
\\
0 \\
0 \\
0 \\
\\
\\
\end{tabular} & 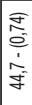 & 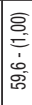 & \begin{tabular}{|l|}
\multirow{2}{*}{} \\
0 \\
0 \\
0 \\
0 \\
0.0
\end{tabular} & $\begin{array}{l}\text { 太ิ } \\
\hat{b} \\
\dot{b} \\
\stackrel{0}{0}\end{array}$ & 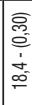 & $=$ & 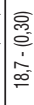 & 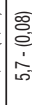 & 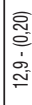 & 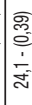 & 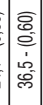 & 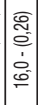 & \begin{tabular}{|c|}
$\overrightarrow{8}$ \\
0 \\
0 \\
$\dot{0}$ \\
$\dot{\sigma}$ \\
\end{tabular} & 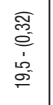 & 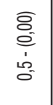 & $\begin{array}{l}E \\
E \\
0 \\
0 \\
0 \\
0 \\
0\end{array}$ & $\begin{array}{l}\bar{o} \\
0 \\
0 \\
\dot{\alpha} \\
0 \\
0\end{array}$ & 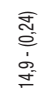 \\
\hline & હે & 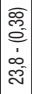 & 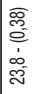 & 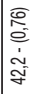 & 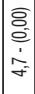 & 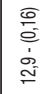 & \begin{tabular}{|l}
$\bar{g}$ \\
0 \\
0 \\
0 \\
0 \\
0 \\
0
\end{tabular} & $\mid \begin{array}{l}0 \\
\bar{\delta} \\
0 \\
\dot{0} \\
\dot{b} \\
\text { s. }\end{array}$ & 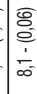 & 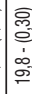 & 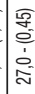 & 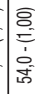 & 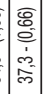 & 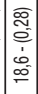 & 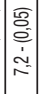 & 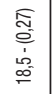 & $\begin{array}{l}\bar{g} \\
\bar{o} \\
\frac{1}{\sigma} \\
\end{array}$ & $\begin{array}{c}\overline{\overline{\hat{\sigma}}} \\
\underline{0} \\
\dot{j} \\
\underline{\sigma}\end{array}$ & $\begin{array}{l}\text { क्ष } \\
0 \\
0 \\
0 \\
0 \\
0\end{array}$ & 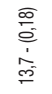 \\
\hline & 崩 & 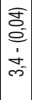 & 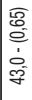 & \begin{tabular}{|l}
0 \\
$\delta$ \\
0 \\
0 \\
0 \\
0 \\
0
\end{tabular} & 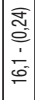 & 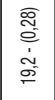 & 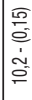 & 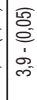 & 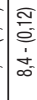 & $\frac{\pi}{\frac{\pi}{3}}$ & 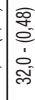 & 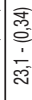 & 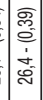 & 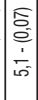 & & 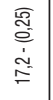 & $\begin{array}{c}\bar{g} \\
0 \\
\dot{0} \\
\dot{\sigma}\end{array}$ & $\begin{array}{l}\bar{g} \\
0 \\
0 \\
\dot{0} \\
\dot{0}\end{array}$ & $\begin{array}{l}\bar{g} \\
o \\
0 \\
\dot{b} \\
\sigma\end{array}$ & $\begin{array}{l}\text { g, } \\
0 \\
\dot{0} \\
\dot{d} \\
0\end{array}$ \\
\hline \multirow{4}{*}{ 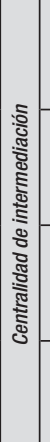 } & 音 & $\mid$\begin{tabular}{|c|}
$\frac{0}{0}$ \\
$\frac{0}{0}$ \\
$\frac{1}{m}$ \\
\end{tabular} & 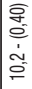 & 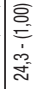 & $\left|\begin{array}{l}\hat{E} \\
\dot{0} \\
\dot{0} \\
\tilde{N}\end{array}\right|$ & $\begin{array}{l}\bar{E} \\
\underline{0} \\
\dot{0} \\
\dot{m} \\
m_{0}\end{array}$ & $\begin{array}{l}\text { s. } \\
0 . \\
\dot{0} \\
-\end{array}$ & $\begin{array}{l}\overline{8} \\
0 \\
\dot{0} \\
\dot{0}\end{array}$ & 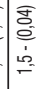 & \begin{tabular}{|l}
$\overline{\bar{D}}$ \\
0 \\
0 \\
0 \\
in
\end{tabular} & $\mid \begin{array}{l}\overline{0} \\
0 \\
0 \\
\dot{0} \\
\dot{\sigma}\end{array}$ & 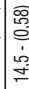 & 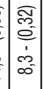 & $\begin{array}{l}0 \\
0 \\
0 \\
0 \\
0 \\
0 \\
0\end{array}$ & 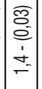 & $\begin{array}{l}\bar{s} \\
\bar{c} \\
\dot{1} \\
\hat{m}\end{array}$ & $\begin{array}{l}\bar{g} \\
0 \\
0 \\
\dot{b} \\
\dot{\delta}\end{array}$ & $\begin{array}{l}\sqrt{8} \\
0 \\
0 \\
\vdots \\
=\end{array}$ & $\begin{array}{l}\text { 总 } \\
0 \\
\vdots \\
\stackrel{-}{=}\end{array}$ & $\begin{array}{l}\bar{E} \\
\overline{0} \\
\dot{\sim} \\
\tilde{n}\end{array}$ \\
\hline & 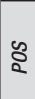 & 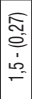 & 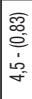 & 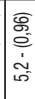 & \begin{tabular}{|c|}
$\mathbf{g}$ \\
0 \\
$\dot{0}$ \\
$\dot{b}$ \\
0
\end{tabular} & 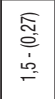 & 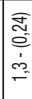 & $\begin{array}{l}\bar{\sigma} \\
\text { o. } \\
\dot{0} \\
\tilde{\sigma}\end{array}$ & 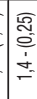 & 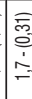 & 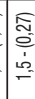 & 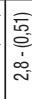 & 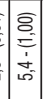 & 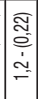 & & 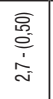 & \begin{tabular}{|l|} 
\\
\\
0 \\
0 \\
$\dot{0}$ \\
0
\end{tabular} & \begin{tabular}{|l|} 
\\
\\
0 \\
0 \\
0 \\
0 \\
0
\end{tabular} & 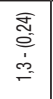 & 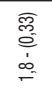 \\
\hline & ผे & 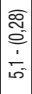 & 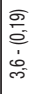 & 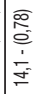 & $\mid \begin{array}{c}\mathbf{s} \\
0 \\
0 \\
0 \\
0 \\
0 \\
\end{array}$ & 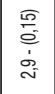 & 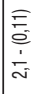 & $=\frac{1}{8}$ & $\mid \begin{array}{l}\overline{8} \\
0 \\
0 \\
0 \\
0\end{array}$ & 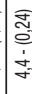 & 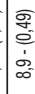 & 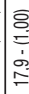 & 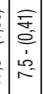 & 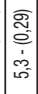 & 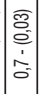 & $\begin{array}{l}\frac{\overline{0}}{0} \\
\frac{1}{m} \\
\frac{1}{m}\end{array}$ & $\begin{array}{l}\bar{g} \\
0 \\
0 \\
0 \\
0 \\
=\end{array}$ & $\begin{array}{l}\text { og } \\
o \\
\dot{0} \\
6 \\
=\end{array}$ & $\begin{array}{l}\bar{g} \\
0 \\
0 \\
0 \\
0 \\
=\end{array}$ & 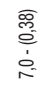 \\
\hline & 岕 & 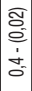 & 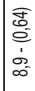 & 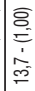 & 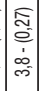 & $\begin{array}{l}\bar{E} \\
\bar{E} \\
\vdots \\
=\end{array}$ & 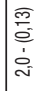 & 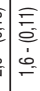 & 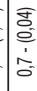 & 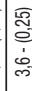 & 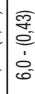 & 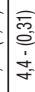 & 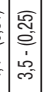 & 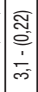 & 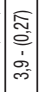 & $\begin{array}{l}\bar{\rho} \\
\overline{0} \\
\dot{0} \\
i \\
\text { ind }\end{array}$ & $\begin{array}{c}\bar{\delta} \\
\overline{0} \\
\dot{1} \\
\frac{1}{0}\end{array}$ & $\begin{array}{l}\bar{\sigma} \\
\overline{0} \\
0 \\
b \\
0 \\
0\end{array}$ & $\begin{array}{l}\bar{g} \\
0 \\
0 \\
b \\
0\end{array}$ & $\begin{array}{l}\bar{g} \\
\dot{0} \\
\dot{0} \\
\stackrel{-}{-}\end{array}$ \\
\hline \multirow{5}{*}{ 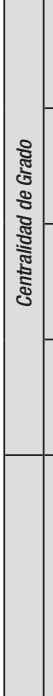 } & $\vec{\Sigma}$ & 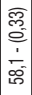 & 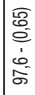 & 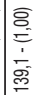 & 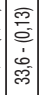 & 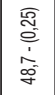 & 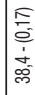 & 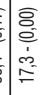 & 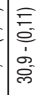 & 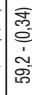 & $\begin{array}{l}\frac{0}{0} \\
\frac{0}{2} \\
\frac{\tilde{c}}{\sigma}\end{array}$ & 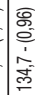 & 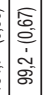 & 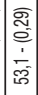 & 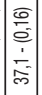 & 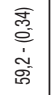 & 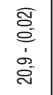 & 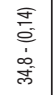 & $\begin{array}{l}\bar{s} \\
0 \\
0 \\
0 \\
\tilde{n} \\
\tilde{n}\end{array}$ & 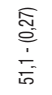 \\
\hline & ర్2 & 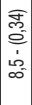 & 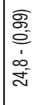 & 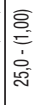 & $\mid \begin{array}{c}\widetilde{\alpha} \\
0 \\
\vdots \\
\vdots \\
\infty \\
\infty \\
\infty\end{array}$ & $\begin{array}{l}\vec{g} \\
0 \\
0 \\
b \\
\stackrel{0}{0}\end{array}$ & 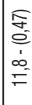 & 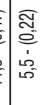 & 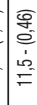 & 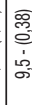 & $\mid \begin{array}{l}\bar{g} \\
0 \\
0 \\
\vdots \\
\sigma \\
\sigma\end{array}$ & 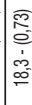 & 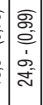 & 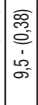 & 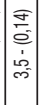 & 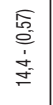 & $\begin{array}{l}\bar{g} \\
o \\
0 \\
\dot{o} \\
o\end{array}$ & 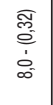 & $\begin{array}{l}\widetilde{\sigma} \\
\tilde{m} \\
\dot{\vdots} \\
\vdots \\
\infty\end{array}$ & $\begin{array}{l}\text { 香 } \\
0 \\
\dot{0} \\
0 \\
=\end{array}$ \\
\hline & के & 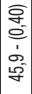 & 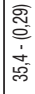 & $\mid \begin{array}{c}\delta \\
0 \\
0 \\
0 \\
\vdots \\
0 \\
0\end{array}$ & $\begin{array}{c}\bar{\sigma} \\
0 \\
0 \\
\dot{a} \\
\sigma \\
\sigma\end{array}$ & 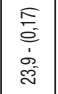 & \begin{tabular}{|l} 
\\
\\
0 \\
0 \\
$\dot{0}$ \\
0 \\
0
\end{tabular} & 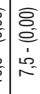 & 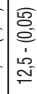 & 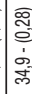 & 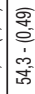 & 总 & $\mid \begin{array}{c}1 \\
0 \\
0 \\
0 \\
0 \\
0 \\
0 \\
0\end{array}$ & 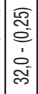 & 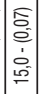 & 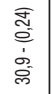 & $\begin{array}{l}\bar{g} \\
0 \\
0 \\
b \\
0 \\
0\end{array}$ & $\begin{array}{l}\bar{\sigma} \\
\bar{\sigma} \\
0 \\
0 \\
0 \\
\alpha \\
\text { s. }\end{array}$ & 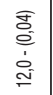 & 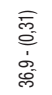 \\
\hline & 岕 & 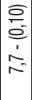 & 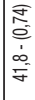 & 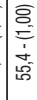 & 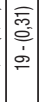 & $\begin{array}{l}\bar{\Phi} \\
0 \\
0 \\
\dot{0} \\
0 \\
0\end{array}$ & 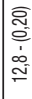 & 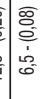 & 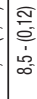 & 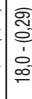 & 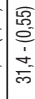 & 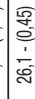 & 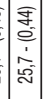 & 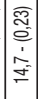 & 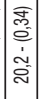 & 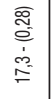 & 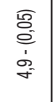 & $\begin{array}{l}\bar{g} \\
0 \\
\dot{0} \\
\dot{\sigma} \\
\text { i }\end{array}$ & $\begin{array}{l}\bar{s} \\
0 \\
0 \\
\vdots \\
0 \\
0\end{array}$ & $\begin{array}{l}\widehat{E} \\
\overline{0} \\
\dot{\vdots} \\
\dot{\infty}\end{array}$ \\
\hline & & o & 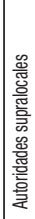 & 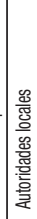 & & 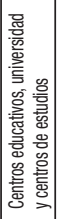 & & 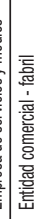 & 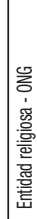 & 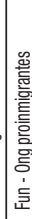 & 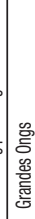 & 穿 & 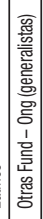 & & 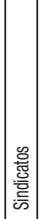 & 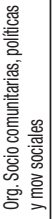 & 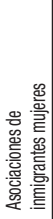 & 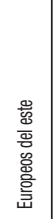 & 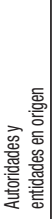 & 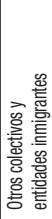 \\
\hline
\end{tabular}

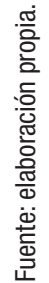


Tal como se indica en la tabla 2, los valores de los indicadores calculados se exponen tanto como puntuaciones normalizadas y, también, como valores estandarizados (entre paréntesis); esto último es una transformación propia que nos facilita la discriminación de los valores más significativos según la distribución propia de los puntajes en cada indicador. Bajo esta definición hemos considerado como relevante todos los indicadores que obtengan valores estandarizados cercanos, iguales o superiores a 0,5.

En función de lo anterior, podemos evidenciar que los actores con puntuaciones más altas son unos pocos y conocidos. Así, desde los actores políticos-administrativos tenemos que los más relevantes son las «autoridades locales» (ayuntamientos principalmente), y también las «autoridades supralocales» (principalmente autonómicas y nacionales). Por otro lado, en el sector civil español, evidenciamos un rol significativo de las Grandes ONGs (con presencia en todo el territorio nacional), las fundaciones y ONGs generalistas (con presencia en territorios concretos), así como las asociaciones de inmigrantes latinos por el lado de actores no nativos. Si los actores anteriores pueden ser definidos como relevantes, hay otros, con menor volumen relacional, que igualmente alcanzan una relevancia media, en tanto cierta significatividad en algunos indicadores, principalmente asimetría positiva, pero poco más. El resto de actores se definen como no relevantes ya que el volumen de sus relaciones para los distintos tipos de indicadores no alcanza una frecuencia tal que permita diferenciarlos con alguna relevancia. El detalle de todos los grupos se muestra en la siguiente tabla.

Dado que son los actores «muy relevantes» son los que alcanzan el mayor peso en el entramado reticular serán estos, entonces, los que establezcan, dados su patrones relacionales, las bases a partir de las cuales definir los distintos roles que buscamos evidenciar. Para esto, y en función de los datos de la tabla 4, podemos esquematizar y resumir los principales patrones relacionales, tal como se muestra en la siguiente tabla: 


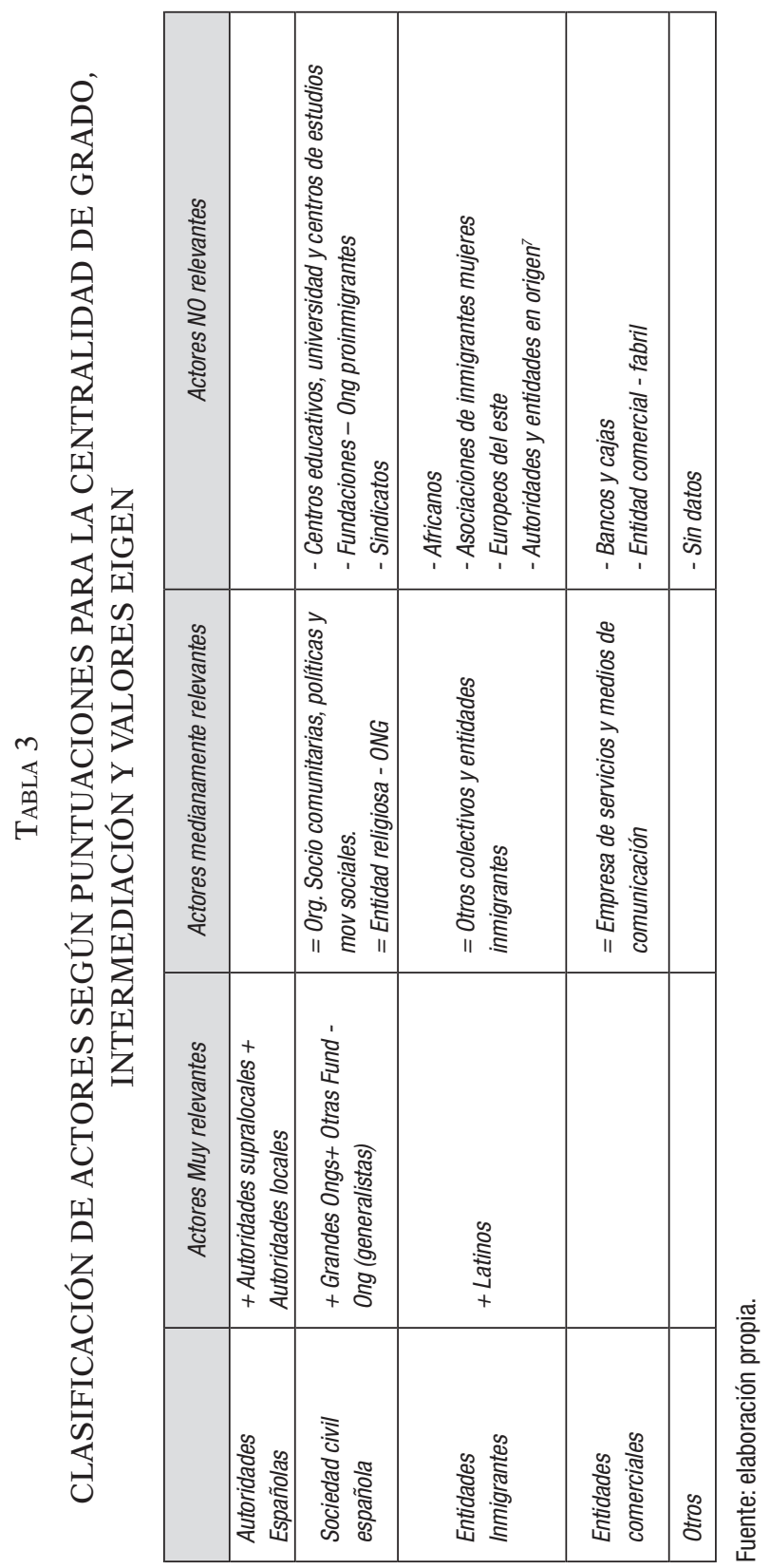

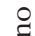

D

궁

.

$\frac{0}{0}$

:

ए

궁

ฮิ

ซี

중

$\frac{0}{2}$

:

สี

훙

$=$

s)

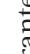

.

.

o

남

ํํㅇ

밍

욤

ส

맘 
Dado que son los actores «muy relevantes» son los que alcanzan el mayor peso en el entramado reticular serán estos, entonces, los que establezcan, dados su patrones relacionales, las bases a partir de las cuales definir los distintos roles que buscamos evidenciar. Para esto, y en función de los datos de la tabla 4, podemos esquematizar y resumir los principales patrones relacionales, tal como se muestra en la siguiente tabla:

TABLA 4

CARACTERIZACIÓN DE LOS ACTORES RELEVANTES SEGÚN LA RELEVANCIA DE CADA TIPO DE INDICADOR PARA CADA TIPO DE RELACIÓN

\begin{tabular}{|l|c|c|c|c|c|c|c|c|c|c|}
\hline & \multicolumn{3}{|c|}{ Centralidad de Grado } & \multicolumn{3}{|c|}{ Valores Eigen } & \multicolumn{3}{|c|}{ Intermediación } \\
\hline \multicolumn{1}{|c|}{ Actores Muy relevantes } & Neg & SYM & Pos & Neg & SYM & Pos & Neg & SYM & Pos \\
\hline + Autoridades supralocales & + & & + & + & & + & + & & + \\
\hline + Autoridades locales & + & + & + & + & + & + & + & + & + \\
\hline+ Grandes Ongs & + & + & & + & + & & & + & \\
\hline + Otras Fund - Ong (generalistas) & & + & + & & + & + & & & + \\
\hline + Latinos & + & + & + & & + & & & + & + \\
\hline
\end{tabular}

La anotación «+» indica una alta relevancia en cada indicador para cada tipo de red. / Fuente: elaboración propia.

Tal como se puede observar, los patrones relacionales más significativos para estos actores cobran una similitud bastante evidente tanto para los valores de centralidad de grado como para los valores eigen, y sólo para los latinos se evidencia cierta disparidad para ambos indicadores. Por otro lado, para los valores de centralidad de intermediación se observa que para ambos tipos de autoridades los patrones son similares. Sin embargo, para los actores de la sociedad civil, sea nativa como inmigrante, las puntuaciones de intermediación siguen distribuciones diferenciadas.

En el caso de las «autoridades locales» la relevancia de sus patrones relacionales se funda en que desarrollan todas las relaciones posibles de forma muy significativa respecto del resto de actores. Principalmente se relacionan, de forma directa, con la mayoría de las asociaciones de inmigrantes a partir de relaciones simétricas $(65,0)^{6}$ y; levemente menos, a través de relaciones asimétricas negativas $(55,4)$, tal como se puede evidenciar en la comparativa de subredes de la figura 3.

6 Se hace referencia al valor estandarizado de la tabla 2. 


\section{FIgURA 3}

SUB RED DE ENTRE AUTORIDADES NATIVAS Y TIPOS DE ASOCIACIONES SEGÚN RELACIONES SIMÉTRICAS Y ASIMÉTRICAS NEGATIVAS

a) Sub red de relaciones simétricas

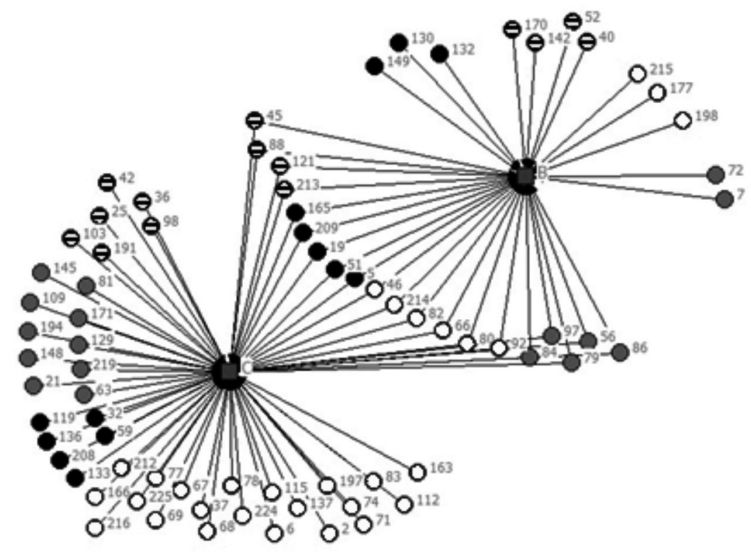

b) Sub red de relaciones asimétricas negativas

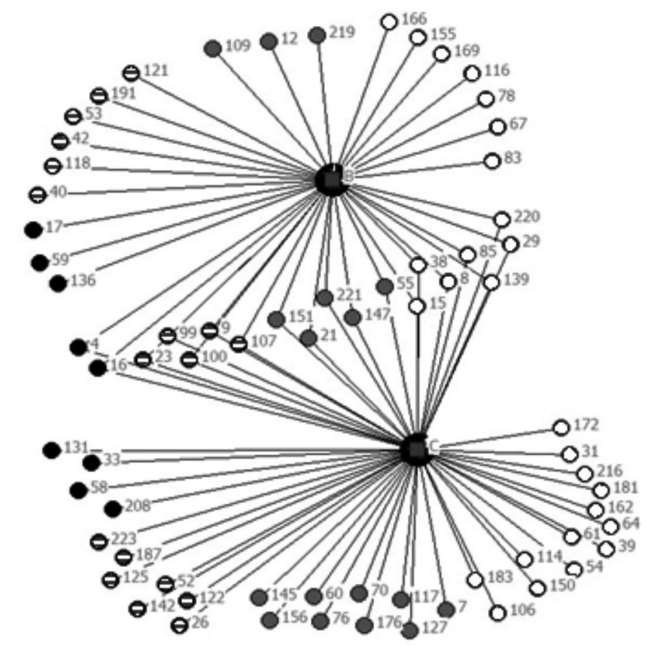

Fuente: elaboración propia.

Nodo blanco $=$ Asociaciones Latinas $/$ Nodo rayado $=$ Asociaciones plurinacionales $/$

Nodo plomo $=$ Asociaciones Africanas $/$ Nodo negro $=$ Asociaciones de Europa del este y ExURSS 
Esta relevancia relacional es un patrón que se repite, cambiando el orden, en los valores eigen, en tanto se dan de forma significativa los tres tipos de relaciones, aunque en este caso las puntuaciones simétricas son las más bajas $(42,2)$, incluso respecto de las de asimetría positiva $(59,6)$. Esto podría estar indicando que las autoridades locales recrean relaciones directas de tipo simétricas con asociaciones de inmigrantes y más asimétricas en términos estructurales, sobre todo más negativas que positivas. En lo que respecta a su papel de conexión de las asociaciones a la red se observa que tanto las relaciones simétricas como las asimétricas cobran una relevancia similar, y con un significativo nivel de asimetría positiva. Por tanto, todo esto implica que el papel relacional que juegan estos actores es polifuncional $\mathrm{y}$, a la vez, contradictorio.

Parte de lo descrito también se hace extensible para los actores, también relevantes, y que hemos denominados como "Autoridades supralocales». Particularmente, la distribución de las relaciones más significativas se distribuyen entre las asimetría negativa $(41,8)$ y también, aunque con algo menos de relevancia, en la asimetría positiva $(24,8)$. A tal punto llega esta polaridad, que los indicadores bajo lógicas simétricas no alcanzan significatividad por si misma y sólo superan a la asimetría positiva en la centralidad de grado. Incluso, la polaridad se expresa en la centralidad de intermediación, o sea, la función de conectar actores a la red se propicia desde la pasividad o asimetría positiva, en tanto son las asociaciones de inmigrantes las que se conectan a la red controlando la relación diádica o, por el contrario, la conexión a la red se define a partir del control que las autoridades ejercen en los términos de la relación. Por tanto, el rol de estos actores, a diferencia de las autoridades españolas locales, se funda en la polaridad de los tipos de relaciones, y siempre con escasa frecuencia para desarrollar relaciones horizontales con las asociaciones de inmigrantes.

En lo que respecta al segundo grupo de actores definidos como "muy relevantes», los de la sociedad civil, observamos que las "grandes ONGs» (Cruz roja, Cáritas, Accem, etc.) desarrollan relaciones directas con las asociaciones de tipo simétrico (54,3) (Fig. 4), aún considerando que las relaciones de tipo asimétricas negativas alcanzan relevancia propia $(31,4)$. A nivel estructural la tendencia se invierte, ya que las relaciones asimétricas negativas $(32,0)$ cobran mayor relevancia que las simétricas $(27,0)$. Aunque esto puede 
suponer que desarrollan un patrón similar a la autoridades locales, cabe destacar que para este caso existe una evidente ausencia de valores significativos referidos a las relaciones asimétricas positivas, lo que supone que las asociaciones de inmigrantes tienen, para estas relaciones, un menor margen de maniobra para controlar las actividades y acciones que emprenden junto a esos actores; más bien, el actuar de estas ONGs es activo y participativo en las relaciones contraídas.

El resto de actores del tercer sector con puntuaciones significativas son las «otras fundaciones y ONGs» de tipo generalistas que, a diferencia del resto de actores analizados, no comparte patrones relacionales significativos que se fundamenten en lógicas de asimetría negativa, alcanzado solo una eventual significatividad en las relaciones directas o centralidad de grado (25,7) (Fig. 4). Por tanto, se evidencia una clara vocación de estos actores por generar y desarrollar relaciones simétricas $(61,6)$, tanto a nivel directo con las asociaciones (centralidad de grado) como a nivel estructural o centralidad de intermediación $(37,3)$. Si cabe destacar que estos actores desarrollan un significativo patrón relacional bajo lógicas de asimetría positiva $(24,9)$, incluso es mayor que la asimetría negativa cuando el foco se pone a nivel estructural (valores eigen) $(36,5)$. A pesar de esto, el repaso general de los indicadores nos refleja el claro rol integrador de estos actores en las distintas dimensiones, sobre todo a nivel de intermediación (aunque bajo a nivel nominal, es el valor más alto de la distribución), lo que indica que las asociaciones con menores conexiones a la red encuentran en estos actores caminos de conexión a la red bajo relaciones cooperativassimétricas $(5,4)$. 
FiguRA 4

SUB RED DE ENTRE ONGS Y TIPOS DE ASOCIACIONES SEGÚN RELACIONES SIMÉTRICAS Y ASIMÉTRICAS NEGATIVAS

a) Sub red de relaciones simétricas

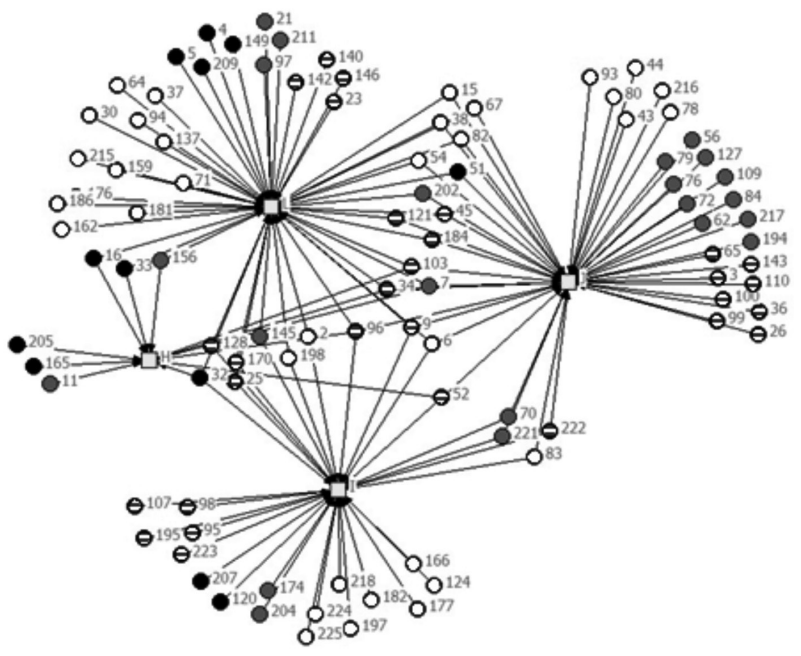

b) Sub red de relaciones asimétricas negativas

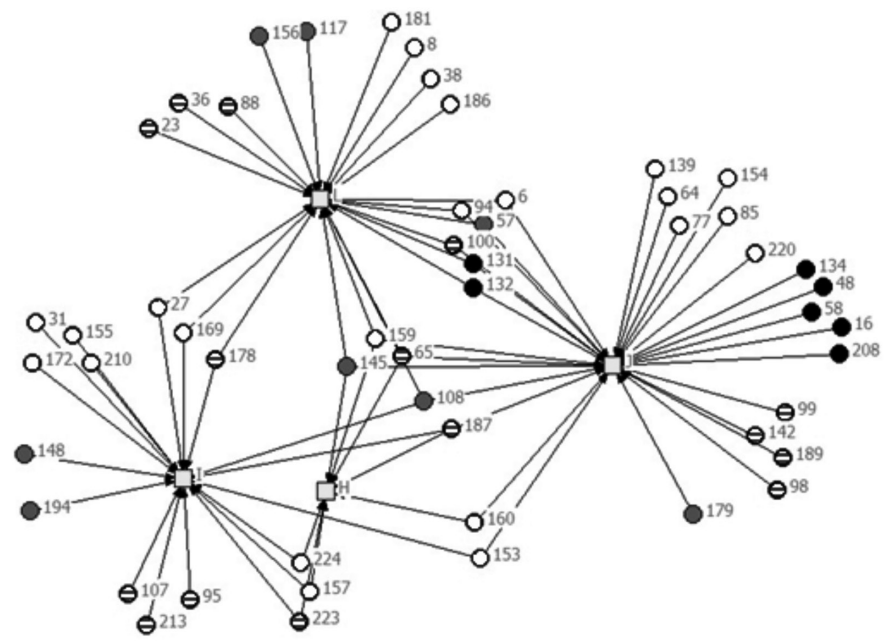


c) Sub red de relaciones asimétricas positivas

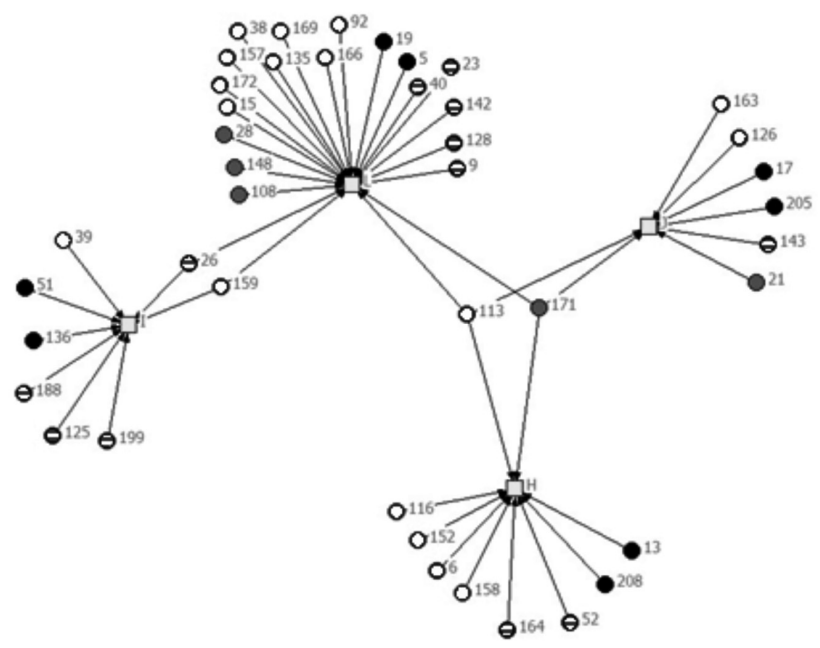

Fuente: elaboración propia.

Por último, y en lo que respecta al papel que las propias asociaciones de inmigrantes juegan como contrapartes de otras asociaciones de inmigrantes, podemos ver que el conjunto de asociaciones latinas es el que, de lejos, juega un papel preponderante y significativo. Esto se explica, en un primer lugar, por la mención transversal que el conjunto de asociaciones y colectivos hace respecto a este tipo de asociaciones, y también, porque el propio colectivo de asociaciones inmigrantes latinas define un patrón relacional altamente simétrico $(102,0)$ y denso con sus pares de origen, algo que se evidencia de forma muy clara en el siguiente gráfico reticular, en tanto que una buena parte de las asociaciones de inmigrantes latinos (nodos blancos) se reúnen en torno a un nodo en particular (Aso. Latinas) que representa a las mismas asociaciones, pero en este caso como contrapartes. 
FigURA 5

SUB RED DE ENTRE ASOCIACIONES (CONTRAPARTES) Y TIPOS DE ASOCIACIONES SEGÚN RELACIONES SIMÉTRICAS Y ASIMÉTRICAS NEGATIVAS

a) Sub red de relaciones simétricas

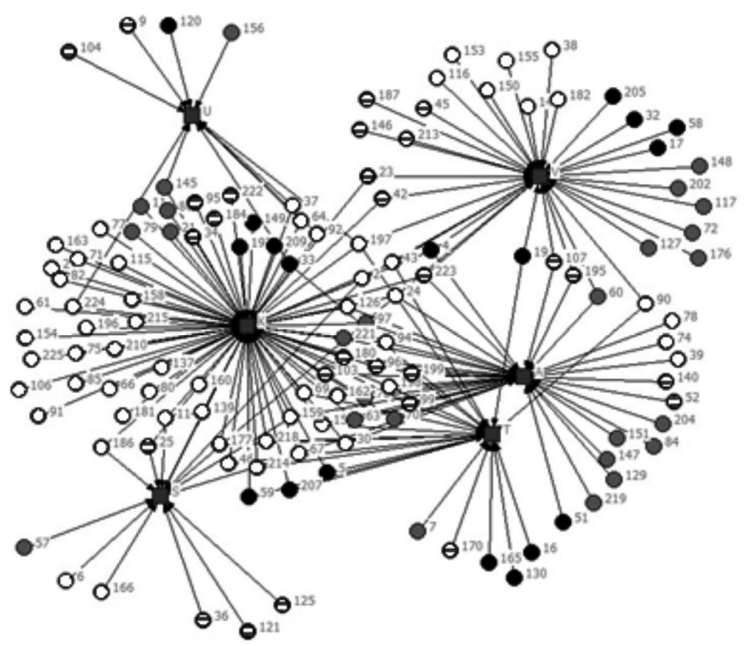

a) Sub red de relaciones asimétricas negativas

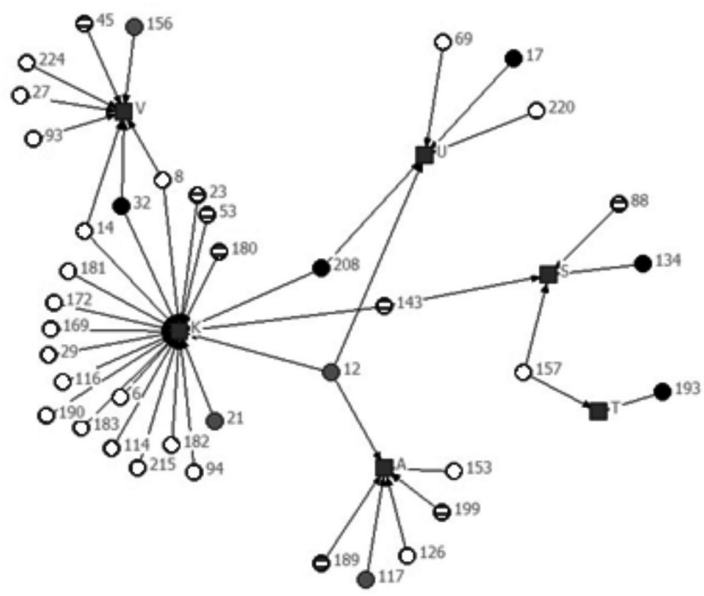

Fuente: elaboración propia. 
Más allá de esta particularidad, las asociaciones latinas, en tanto contrapartes, fundamentan con claridad su patrón relacional simétrico tanto a nivel directo (centralidad de grado) como a nivel estructural (valores eigen), incluso la cierta relevancia de la asimetría positiva no logra distorsionar este claro rol «cohesivo» de las asociaciones como contrapartes, y aún más cuando el patrón relacional se evalúa en torno a la centralidad de intermediación, donde alcanzan la puntuación más alta en la relaciones de simetría. Esto reafirma este papel cohesivo, es decir, la puerta de entrada de otras asociaciones a la red de actores. Bajo esta lógica, el resto de asociaciones de inmigrantes, en tanto contrapartes, si bien no evidencian patrones relacionales tan contundentes como en el caso latino, si desarrollan roles relacionales similares, lo que permite afirmar, de alguna manera, que el conjunto de las contrapartes inmigrantes juegan un papel eminentemente cohesivo con sus pares connacionales.

Respecto del segundo grupo de actores, los que denominamos «medianamente relevantes» vemos que, para todos ellos, su clasificación se fundamenta al presentar un único patrón relacional significativo, esto es, la relevancia de sus relaciones asimétricas positivas, particularmente para el caso de la centralidad de grado. A destacar dentro de esta categoría se encuentran actores de distintos grupos: sociedad civil, otras organizaciones inmigrantes, entidades comerciales y religiosas. Es probable que, dado el perfil de estos actores (Tabla 3), la asimetría positiva responda a que las relaciones que preferentemente desarrollan este tipo de actores se inscriben en lógicas de tipo pasivo o donde no se recrean o proyectan actividades que conlleven un trabajo conjunto: relaciones comerciales con las empresas, asistencialistas desde las entidades religiosas, soporte o información desde los consulados y/o federaciones, etc. de ahí que se pueda, eventualmente, encontrar una explicación común para entender este tipo de patrones y roles.

En general, los actores más relevantes definen patrones relacionales, tanto a nivel local como general, bastante similares, por tanto, tomaremos la centralidad de grado como un indicador que resuma y que permita la comparación entre los actores, sumando a esto consideraremos también la relevancia de los patrones estructurales de conexión o centralidad de intermediación. A partir de lo anterior, presentamos un gráfico de dispersión (Fig. 6) con una triple intención, primero intentamos resumir el análisis hasta aquí descrito, también pretendemos integrar en dicho análisis al resto de contrapartes que dada su escasa relevancia no han sido 
mencionadas en detalle y, finalmente, evaluar el volumen o peso de cada actor en función su indicadores. Para todo esto hemos definido la posición de todas las contrapartes en un gráfico de dos coordenadas y según tres criterios: El primero se representa en el eje horizontal según la diferencia entre la centralidad de grado (normalizado) obtenido en la red simétrica y la centralidad de grado obtenido en la red asimétrica negativa. El segundo criterio se representa en el eje vertical según la diferencias entre la centralidad de intermediación (normalizada) obtenida en la red simétrica y la red asimétrica negativa ${ }^{7}$. Finalmente, el tamaño de las figuras nos habla del volumen relacional de cada contraparte, asumiendo que dicho volumen o frecuencia es un factor a considerar a la hora de representar las diferencias. Por último, a efectos de construcción de la tipología, aparecen sombreados los actores de naturaleza inmigrante.

Tal como podemos observar, se evidencia con claridad lo descrito para las contrapartes más relevantes (con mayor tamaño), y a la vez, la posición del resto de entidades evaluadas. De este modo, podemos distinguir con claridad ciertos grupos funcionales (diferenciados por formas y tonalidades) que dan cuenta de distintos roles relacionales. Así, en el sector izquierdo bajo de la gráfica (círculos) se ubican los actores con diferenciales negativas para ambos indicadores. De estos actores destacamos a las Autoridades supralocales como principal exponente de un patrón relacional asimétrico tanto de intermediación como de relaciones directas, lo que supone un claro rol "no integrador» en sus relaciones con las asociaciones de inmigrantes. El siguiente grupo (triángulos), se define porque detentan un diferencial relacional positivo, aunque escaso, pero sobre todo, porque desarrollan sus relaciones de intermediación bajo lógicas de control, lo que supone un perfil conexión asimétrica. Por último, el resto de actores cumplen, en general, roles más bien integradores, tanto por el saldo positivo de centralidad de grado como de intermediación. No obstante lo anterior, dentro de estos existen, al menos, dos grupos diferenciados. Por un lado el grupo de los que obtienen diferenciales exiguos y cercanos a 0 o posicionalmente

7 No incluimos la asimetría positiva ya que, por un lado, su relevancia resulta escasa y, por otro, su inclusión en el gráfico sólo aumentaría innecesariamente la complejidad del mismo de cara a evaluar los roles en función de los estadios de integración social. 
Figura 6

GRÁFICO DE DISPERSIÓN DE LOS TIPOS DE ACTORES

SEGÚN EL SALDO ENTRE LAS PUNTUACIONES DE

CENTRALIDAD DE INTERMEDIACIÓN Y DE GRADO

SIMÉTRICA RESPECTO DE LAS PUNTUACIONES

ASIMÉTRICAS NEGATIVAS

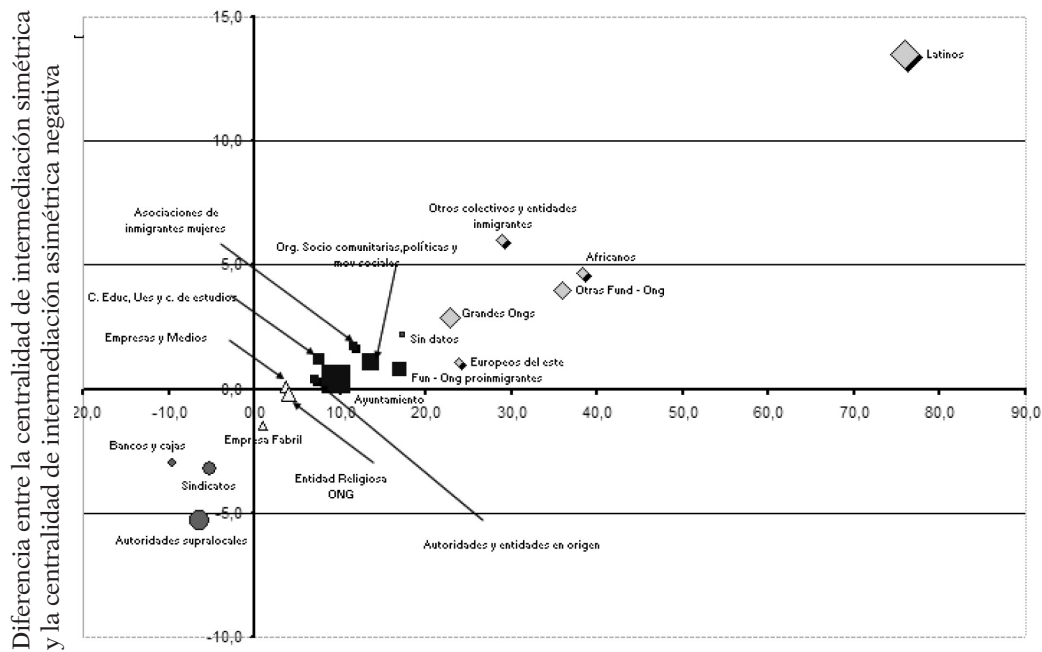

Diferencia entre la centralidad de grado simétrica y la centralidad de grado de asimétrica negativa

Fuente: elaboración propia.

cercanos a la abscisa (cuadrados) y que desarrollan perfiles que podrían ser denominados como de «baja integración». Por el contrario, otro grupo de actores (rombos), alcanzan diferenciales positivos y considerables tanto a nivel de centralidad de grado y, en algunos casos, de intermediación, lo que en su conjunto indica que son actores que pueden ser clasificados como contrapartes que desarrollan «importantes o relevantes» funciones integradoras, tal es el caso, por ejemplo, de las «Otras fundaciones y ONGs» por parte de los actores nativos, y las asociaciones Latinas por parte de la sociedad civil inmigrante. 


\section{CONCLUSIONES}

A la vista de los datos analizados es posible evidenciar los distintos roles que cumplen los actores del entorno en el entramado relacional que tejen junto a las asociaciones de inmigrantes.

Un primer elemento a considerar está en la comparación de volúmenes relacionales, donde vemos que se plantean diferencias importantes, sea esto según su capacidad de relacionarse y/o según el rol o patrón relacional que desempeñen. Así, por ejemplo, el volumen relacional desarrollado por las autoridades locales no es igual que, por poner un ejemplo, el que desarrollan las entidades y ONGs religiosas, aunque en ambas prevalezca un patrón relacional que tiende hacia la simetría relacional. Por ello, una primera conclusión es la evidente significatividad diferenciada que algunos actores alcanzan sobre otros y, desde ello, las posibilidades de integración que cada uno oferta a las asociaciones inmigrantes. En función de todo lo anterior y lo presentado en los resultados, podemos consolidar los roles de las contrapartes según la frecuencia y patrones relacionales, tal como se evidencia en la siguiente tabla resumen. 


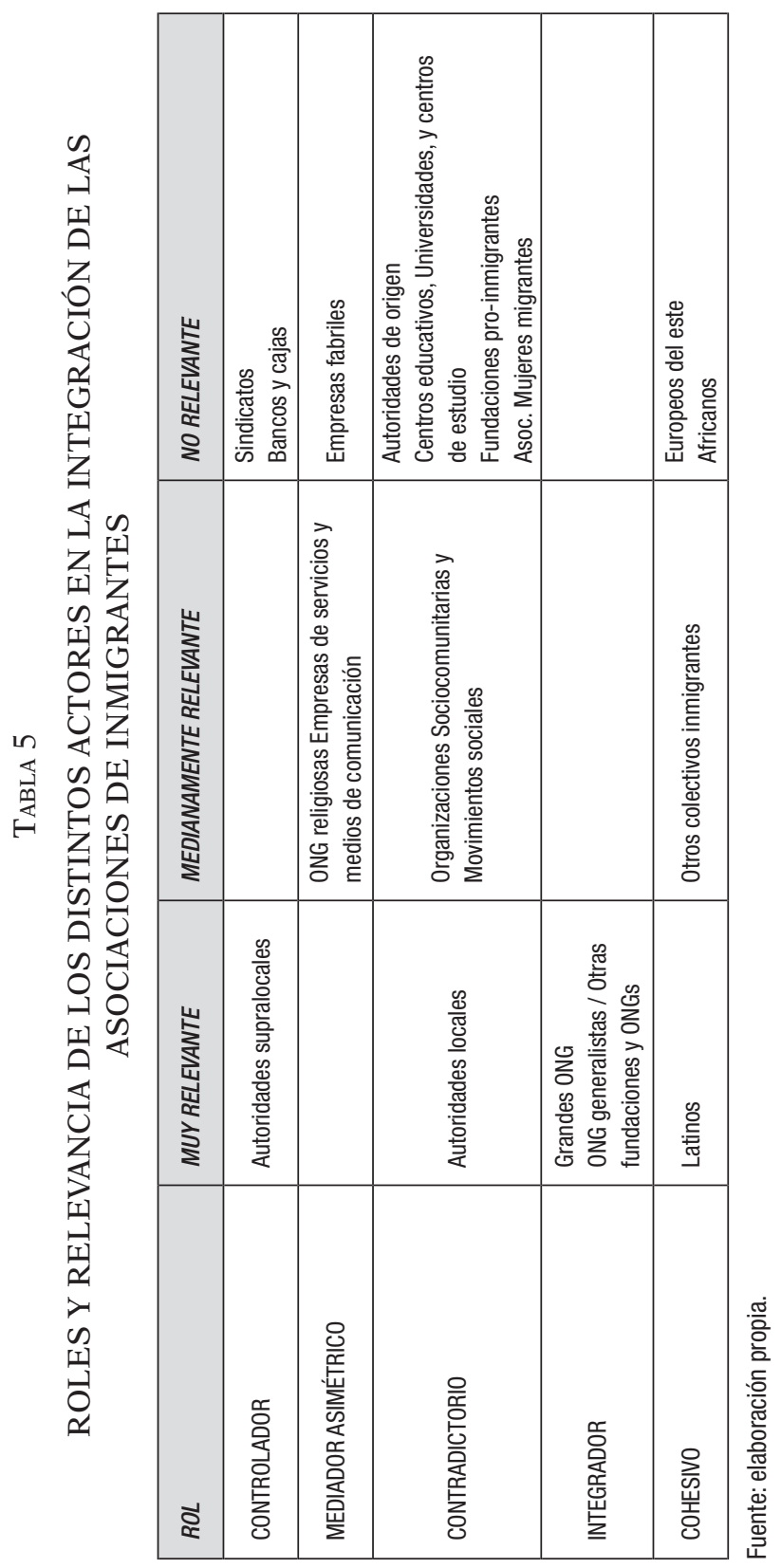


Los roles de las contrapartes con mayor relevancia emergen con cierta claridad. Así, en un primer lugar podemos referirnos al rol que hemos denominado como «controlador» (o excluyente) y lo desarrollan sindicatos, bancos, cajas y, con mayor significatividad y relevancia, las denominadas "Autoridades supralocales», que bajo patrones relacionales predominantemente de control ofrecenoportunidades limitadas para que estos actores inmigrantes se integren en el circuito de relaciones interorganizativas nativas con probabilidades ciertas de emprender sus propias acciones y decisiones. Un breve comentario sobre la ubicación de los sindicatos en este rol puede deberse a que estos actores se enfocan en problemáticas netamente laborales, lo que restringe las posibilidades de las asociaciones para plantear, como contraparte "no experta», sus propias directrices y decisiones; además, no se debe olvidar que ambos actores compiten por el concurso de los sujetos inmigrantes, el primero como trabajadores, el segundo como su base de representación (Veredas, 2000).

Un segundo rol denominado como «intermediador asimétrico» es el que cumplen, con escaso volumen relacional, actores que en gran parte desarrollan un importante número de relaciones pasivas, sin embargo, también alcanzan notoriedad por su escaso diferencial positivo de relaciones simétricas, y sobre todo, por su tendencia a la intermediación bajo el control de las relaciones, lo que supone que su rol de intermediación y conexión de las asociaciones a la red de la sociedad nativa es escaso y, sobre todo, asimétrico. En consecuencia, son actores más bien periféricos en el contexto general, pero que se caracterizan en su actuar por integrar y conectar a las asociaciones bajo relaciones típicamente asimétricas.

Un tercer patrón relacional desempeñado por un grupo considerable de actores fundamenta el rol definido como "contradictorio». $\mathrm{Su}$ principal exponente son las autoridades locales, principalmente los ayuntamientos, que con un elevado volumen relacional se alzan como uno de los actores nativos más preponderantes. La particularidad del rol que desempeñan está en que favorecen tanto patrones relacionales integradores como de control. Esto podría explicarse de cara a las posibilidades dispares que las asociaciones tienen para concordar sus objetivos con los que plantean las autoridades locales que cuentan con intereses, muchas veces, distintos a los de los sujetos inmigrantes, por cuestiones políticas, ideológicas o programáticas, tal y como lo indica el reciente trabajo de Llevot y Garreta cuando afirma que: «Las orientaciones de las 
ayudas públicas al ámbito cultural han sido mencionadas como la clave para comprobar mejor qué actividades se potencian en las asociaciones y cuáles no» (Llevot y Garreta i Bochaca, 2013:186). También se entiende por la necesidad que las asociaciones tienen para acceder a distintos recursos, lo que puede llevar a las asociaciones a que supediten sus intereses en pos de acceder a dichos recursos, algo que se puede observar, con evidente claridad, en las asociaciones que desarrollan acciones y actividades principalmente de tipo políticas reivindicativas que, en caso de estar en contra de los lineamientos de las autoridades locales pueden acabar excluidas de los recursos y subvenciones que convocan estas autoridades (Toral, 2010; Veredas, 2003; Moya, 2005); en un contexto en que las fuentes de financiación locales y directas son fundamentales, y más aún, en época de crisis económica (Garreta i Bochaca y Llevot, 2013).

Por otro lado, las relaciones simétricas pueden explicarse por la necesidad que tienen las autoridades locales de trabajar con los colectivos inmigrados y proporcionar soluciones a sus necesidades o por la simple la externalización de ciertas funciones a estos grupos inmigrantes organizados.

En el ámbito de la sociedad civil, vemos que los roles parecen ser bastante claros para los dos actores más relevantes, esto es, las grandes ONG (presencia nacional) y, sobre todo, las ONG generalistas (presencia no nacional) o definidas como «Otras fundaciones y ONGs», en tanto estos actores desarrollan un rol que podemos denominar como «integrador», sobre todo estas últimas, en tanto alcanzan con claridad una mayor preponderancia en el ámbito de las relaciones simétricas. Este rol puede responder a que las asociaciones de inmigrantes son, la mayor parte de las veces, el objeto de trabajo de las ONGs y, desde ello, resorte de las necesidades de los colectivos desfavorecidos y marginados, lo que permitiría una mayor afinidad de objetivos y posibilidades de participación en las actividades emprendidas de forma conjunta. Sin olvidar, además, que no pocas ONGs se plantean como objetivos prioritarios el fomentar el asociacionismo y la dinamización de las organizaciones de base ciudadana, aunque a veces, desde el paternalismo.(Canales y Zlolniski, 2000; Gil, 2007).

Dentro de este patrón integrador diferenciamos un rol que hemos denominamos como "cohesivo» dado que la función integradora la cumplen actores de naturaleza inmigrante, es decir, son las propias entidades inmigrantes, sobre todo las asociaciones 
latinas, las que como contrapartes integran a otras asociaciones de inmigrantes. Este rol cobra relevancia evidente toda vez que las relaciones inter-asociaciones responden a un sustrato común de sujetos que comparten, en la mayoría de las ocasiones, condiciones, problemáticas y, probablemente, objetivos similares, lo que lleva o facilita la posibilidades de unir fuerzas y recursos para desarrollar actividades mancomunadas definidas bajo lógicas de simetría relacional; a la vez, también son, para el caso de las asociaciones latinas, los principales puntos de contacto para que las asociaciones con menor volumen relacional puedan conectarse a la red nativa, es decir, su rol de intermediación simétrico permite que la integración temprana de las asociaciones a la red interorganizativa de la sociedad de acogida sea bajo condiciones más integradoras que controladoras.

\section{BIBLIOGRAFÍA}

Álvarez de los Mozos, F. (2013): Capital social de las Asociaciones de Inmigrantes: asociaciones bolivianas, colombianas, ecuatorianas y peruanas en Barcelona, Bilbao, Madrid y Valencia. Universidad de Deusto.

Aparicio, R., y Tornos, A. (2010): Las asociaciones de inmigrantes en España. Una visión de conjunto. Madrid, Ministerio de Trabajo y Asuntos Sociales, Subdirección General de Información y Administraciones Públicas.

Arcas, N., y Hernández, M. (2007): «Colaboración de los compradores de las cooperativas agroalimentarias: factores explicativos y efectos sobre el desempeño de la relación». CIRIEC-España, revista de economía pública, social y cooperativa (57), pp.151-171.

BolíbAR, M. (2010): «Redes asociativas, inmigración y participación. Una aproximación empírica a los vínculos entre capital social y participación asociativa.» Págs. 1-26 en Grupo de trabajo 20. Movimientos Sociales, Acción Colectiva y Cambio Social. X Congreso Español de Sociología. Universidad Pública de Navarra, Pamplona.

Borgatti, S. y Everett, M. (1997): «Network analysis of 2-mode data». Social networks, 19(3), pp. 243-269. DOI: 10.1016/S0378-8733(96)00301-2

CANALES, A. y ZlolnisKI, C. (2000): «Comunidades transnacionales y migración en la era de la globalización», ponencia presentada en el Simposio sobre Migración Internacional en las Américas, realizado en San José, Costa Rica, los días 4-6 de septiembre del 2000.

Cheng, A. (2005): «Politics Without the Politics: The Evolving Political Cultures of Ethnic Non-Profits in Koreatown, Los Angeles». Journal of Ethnic and Migration Studies 31(5), pp. 911-929. DOI: 10.1080/13691830500177701 
Coleman, J. (1990): Foundations of Social Theory. Cambridge (Massachusetts), Belknap Press.

FlorA, J. (1998): «Social Capital and Communities of Place» Rural sociology 63 (4), pp. 481-506. DOI: 10.1111/j.1549-0831.1998.tb00689.x

Frey, B., Lohmeier, J., Lee, S. y Tollefson, N. (2006): «Measuring collaboration among grant partners». American Journal of Evaluation 27(3): 383-392.

Fukuyama, F. (2001): «Social capital, civil society and development» Third world quarterly 22 (1), pp. 7-20. DOI: 10.1080/713701144

Fullaondo, A. (2007): "La inmigración en España: Una aproximación metropolitana comparada». Revista ACE (Arquitectura, Ciudad y Entorno) 4, pp. 497-518.

GAJDA, R. (2004): «Utilizing collaboration theory to evaluate strategic alliances». American Journal of Evaluation 25(1), pp. 65-77.

GarnicA, A., Rivero, A. y Ibarra, E. (2004): «La coordinación «híbrida» desde las perspectivas de Williamson y de Nooteboom». Análisis Económico 19(40), pp. 101-117.

Garreta i Bochaca, J. y Llevot, N. (2013): «Las asociaciones de inmigrantes africanos. Organización, proyección y actuaciones». Revista Internacional de Sociología. 71(Extra_1), pp. 15-38.

GIL, A.(2007): La organización de los inmigrantes como potenciador y/o inhibidor de la inserción en las sociedades de destino: el caso de los colombianos en España. AIBR: Revista de Antropología Iberoamericana, 2(3), pp. 521-549.

Gómez, C. (2008): «El asociacionismo de los inmigrantes», dins García Roca, Joaquín i Lacomba, Joan (eds.), La inmigración en la sociedad española, Barcelona, Bellaterra, pp. 541-557.

GonzÁlez, A. y Morales, L. (2006): «Las Asociaciones de Inmigrantes en Madrid: una nota de investigación sobre su grado de integración política.» Revista española del tercer sector (4), pp. 129-174.

Hanneman, R. y Riddle, M. (2005): Introduction to Social Network Methods. Caliornia, University of California.

Hernández-Espallardo, M., y Arcas-Lario, N. (2003): "The effects of authoritative mechanisms of coordination on market orientation in asymmetrical channel partnerships». International Journal of Research in Marketing 20(2), pp. 133-152. DOI: 10.1016/S0167-8116(03)00015-6

Herrera, M. (2008): «Las asociaciones de inmigrantes: un instrumento para la integración». Temas para el debate (160), pp. 50-52.

Kärrholm, J. (2007): Co-operation among rehabilitation actors for return to working life. Stockholm, Sweden, Karolinska Institutet.

LAZEGA, E. (2004): «Racionalidad, disciplina social y estructura». Redes: Revista hispana para el análisis de redes sociales 5(6).

Levine, S. y White, P. (1961): «Exchange as a conceptual framework for the study of interorganizational relationships». Administrative science quarterly 5(4), pp. 583-601. Stable URL: http://www.jstor.org/stable/2390622 
Lozares, C., Roldán, P., Verd, J., Martí, J. y Molina, J. (2011): «Cohesión, Vinculación e Integración sociales en el marco del Capital Social». Redes: Revista hispana para el análisis de redes sociales 20(1):1-28.

Llevot, N., y GarReta i Bochaca, J. (2013): «La mediación intercultural en las asociaciones de inmigrantes de origen africano». Revista internacional de sociología, 71(Extra 1), pp. 167-188.

Mayoux, L. (2001): «Tackling the down side: Social capital, women's empowerment and microfinance in Cameroon»Development and change 32 (3), pp. 435-464. DOI: 10.1111/1467-7660.00212

Morales, L., Anduiza, E., Rodríguez, E., y San Martín, J. (2008): «Capital social, pautas identitarias y actitudes hacia» los otros»: la incorporación cívica de la población de origen inmigrante en Barcelona y Madrid.» Panorama social (8), pp. 119-142.

Morell, A. (2005): «El papel de las asociaciones de inmigrantes en la sociedad de acogida: cuestiones teóricas y evidencia empírica». Migraciones (17), pp. 111-142.

Moya, J. (2005): «Immigrants and associations: a global and historical perspective». Journal of ethnic and migration studies 31(5), pp. 833-864. DOI: $10.1080 / 13691830500178147$

Opsahl, T., Agneessens, F. y Skvoretz, J. (2010): «Node centrality in weighted networks: Generalizing degree and shortest paths». Social Networks 32.3, pp. 245-251. DOI: 10.1016/j.socnet.2010.03.006

Ostrom, E., Ahn, T., y Olivares, C. (2003): «Una perspectiva del capital social desde las ciencias sociales: capital social y acción colectiva» Revista Mexicana de Sociología. 65 (1), pp. 155-233. Stable URL: http://www.jstor.org/ stable/3541518

Plascencia, J. (2005): «Tres visiones sobre capital social: Bourdieu, Coleman y Putnam». Acta republicana: Política y Sociedad 4(4), pp. 21-36.

Portela, M. y Neira, I. (2002): “Capital Social: Concepto y Estudio Econométrico sobre el Capital Social en España». Estudios económicos de desarrollo internacional 2(2), pp. 25-52.

Putnam, R. (1993): Making Democracy Work: Civic Traditions in Modern Italy.

Princeton Univ. Press, Princeton. [Para que la democracia funcione: las tradiciones cívicas en la Italia moderna, CIS, Madrid 2011]

Putnam, R. (1995): «Bowling Alone: America's Declining Social Capital». Journal of Democracy, 6 (1). pp. 65-78. DOI: 10.1353/jod.1995.0002

Putnam, R. (2001): Bowling Alone. New York, Simon and Schuster.

Rodríguez, G., y CARrasco, C. (2003): Las entidades voluntarias de acción social en España Madrid, Cáritas Española.

SARdinha, J. (2005): "Cape Verdean Associations in the Metropolitan Area of Lisbon: their role in integration». Sussex Centre for Migration Research, working paper (26).

Schul, P. y Babakus, E. (1988): «An examination of the interfirm power- conflict relationship: the intervening role of the channel decision structure». Journal of Retailing, 64(4), pp. 381-405. 
Smith, A. (2003): "Civic and citizenship education in contested and divided societies». en International Symposium on National Education. Hong Kong, China.

Tillie, J. (2004): "Social capital of organisations and their members: explaining the political integration of immigrants in Amsterdam». Journal of Ethnic and Migration Studies 30(3), pp. 529-541. DOI: 10.1080/13691830410001682070

Toral, G. (2010): «Las asociaciones de inmigrantes como sociedad civil: un análisis tridimensional». Revista española de investigaciones sociológicas, 132(1), pp. 105-130.

VeredAs, S. (2000): «Sindicatos y asociaciones de inmigrantes una relación difícil pero necesaria». En Morente Mejías (eds.) Cuadernos étnicos: inmigrantes, claves para el futuro inmediato, Jaén. Universidad de Jaén. ISBN 84-8439-027-6, pp. 145-160

VEREDAS, S. (2003): "Las asociaciones de inmigrantes en España». Revista internacional de sociología (36), pp. 207-225. DOI: 10.3989/ris.2003.i36.320

World BANK (1997): Social capital: the missing link?. En Expanding the Measure of Wealth: Indicators of Environmentally Sustainable Development. Washington, The World Bank. 
